\title{
Putting cholesterol in its place: apoE and reverse cholesterol transport
}

\author{
Robert W. Mahley, 1,2,3,4,5 Yadong Huang,,1,2,3,6 and Karl H. Weisgraber1,2,3,5
}

${ }^{1}$ Gladstone Institute of Neurological Disease and ${ }^{2}$ Gladstone Institute of Cardiovascular Disease, San Francisco, California, USA. ${ }^{3}$ Department of Pathology, ${ }^{4}$ Department of Medicine, ${ }^{5}$ Cardiovascular Research Institute, and ${ }^{6}$ Department of Neurology, UCSF, San Francisco, California, USA.

\begin{abstract}
To avoid toxic overload of cholesterol in peripheral cells, the reverse cholesterol transport pathway directs excess cholesterol through HDL acceptors to the liver for elimination. In this issue of the JCI, a study by Matsuura et al. reveals new features of this pathway, including the importance of the ATP-binding cassette transporter G1 in macrophages and apoE in cholesteryl efflux from cells to cholesterol ester-rich (CE-rich) $\mathrm{HDL}_{2}$ acceptors (see the related article beginning on page 1435). One proposal for boosting reverse cholesterol transport has been to elevate plasma HDL levels by inhibiting CE transfer protein (CETP), which transfers CE from HDL to lower-density lipoproteins. However, there has been concern that large, CE-rich $\mathrm{HDL}_{2}$ generated by CETP inhibition might impair reverse cholesterol transport. ApoE uniquely facilitates reverse cholesterol transport by allowing CE-rich core expansion in HDL. In lower species, these large HDLs are not atherogenic. Thus, CETP might not be essential for reverse cholesterol transport in humans, raising hope of using a CETP inhibitor to elevate HDL levels.
\end{abstract}

\section{The reverse cholesterol transport pathway}

While cholesterol is an essential component of mammalian cell membranes, in excess it is toxic to cells. Reverse cholesterol transport is a critical pathway for maintaining cholesterol homeostasis in mammals (1-4). Since the cholesterol ring structure cannot be broken down by cells into carbon dioxide and water, this pathway has evolved to transport excess cholesterol from peripheral cells to the liver for excretion directly into bile or for conversion to bile acids and then excretion.

A number of cellular lipid transporters and receptors and a spectrum of HDL and HDL intermediates participate in this movement of peripheral cholesterol (Figure 1). At one end of the spectrum are lipid-free or lipid-poor apoA-I particles, referred to as pre- $\beta$ HDL. These particles are secreted by the liver and small intestine or generated from surface material from partially lipolyzed chylomicrons or from $\mathrm{HDL}_{2}$ in the periphery by the action

Nonstandard abbreviations used: CE, cholesteryl ester; CETP, CE transfer protein; LCAT, lecithin:cholesterol acyltransferase.

Conflict of interest: The authors have declared that no conflict of interest exists.

Citation for this article: J. Clin. Invest. 116:1226-1229 (2006). doi:10.1172/JCI28632. of cholesteryl ester (CE) transfer protein (CETP), hepatic lipase, or phospholipid transfer protein (5-9). These HDL precursors are acceptors for unesterified cholesterol and phospholipids transferred by the ATP-binding cassette transporter A1 (ABCA1) on peripheral cells, giving rise to discoidal lipoproteins containing apoA-I (for review, see refs. 2, 10, 11). The acquired unesterified cholesterol is esterified by the plasma enzyme lecithin:cholesterol acyltransferase (LCAT). The CE is then packaged into the hydrophobic core of the discoidal particle, converting it to spherical $\mathrm{HDL}_{3}$. $\mathrm{HDL}_{3}$ can continue to accept unesterified cholesterol and phospholipids from the class B, type I scavenger receptor (SR-BI); through continued action of LCAT, the core expands and the particle size increases, forming $\mathrm{HDL}_{2}$. Although the major apolipoprotein com$\mathrm{HDL}_{2}$ also contains significant amounts of apoE $(12,13)$.

The study by Matsuura et al. in this issue of the JCI (14) adds ABCG1 present on macrophages to the list of components that contribute to the formation of $\mathrm{HDL}_{2}$. This is of particular relevance, as monocyte-derived macrophages enter the artery wall, where they scavenge accumulating cholesterol in an attempt to preponent in $\mathrm{HDL}_{3}$ and $\mathrm{HDL}_{2}$ is apoA-I, vent its buildup, leading to an atherosclerotic plaque. Therefore, it is important that cholesterol-loaded macrophages, or foam cells, possess a mechanism to eliminate excess cholesterol before they suffer the toxic effects of this excess.

\section{Differences in reverse cholesterol transport among species}

To complete the transport process from $\mathrm{HDL}_{2}$ to the liver in humans, primates, and a few other species, CE is transferred by CETP to lower-density lipoproteins (Figure 1), which deliver their cholesterol loads through interaction with LDL receptors in the liver. Alternatively, $\mathrm{HDL}_{2}$ can directly transfer CE to hepatic cells via SR-BIs (15). However, many lower species lack CETP (16) and use HDL as their main plasma cholesterol transporters. In CETPpossessing species, LDLs carry the majority of the plasma cholesterol.

In the absence of CETP, how do these species complete reverse cholesterol transport? A major distinguishing characteristic of their HDL is the presence of a prominent subclass that is enriched in apoE, referred to as $\mathrm{HDL}_{1}, \mathrm{HDL}_{\mathrm{c}}$, or $\mathrm{HDL}$-with apoE (for review, see refs. 12, 17, 18). Since apoE is an effective ligand for LDL receptors, these apoE-enriched HDLs can deliver cholesterol acquired from the periphery to the liver via hepatic LDL receptors (19). CETP-deficient species are particularly resistant to diet-induced elevations in plasma cholesterol levels, demonstrating that they possess an effective reverse cholesterol transport pathway in the absence of CETP. They accomplish this through HDL-with apoE. Their high HDL concentrations and resistance to atherosclerosis are also consistent with the inverse correlation between HDL levels and risk of atherosclerosis observed in humans (1).

Concentrations of HDL-with apoE are low in humans $(12,13)$ and are thought to represent a minor component of reverse cholesterol transport. However, in subjects with CETP mutations that lead 


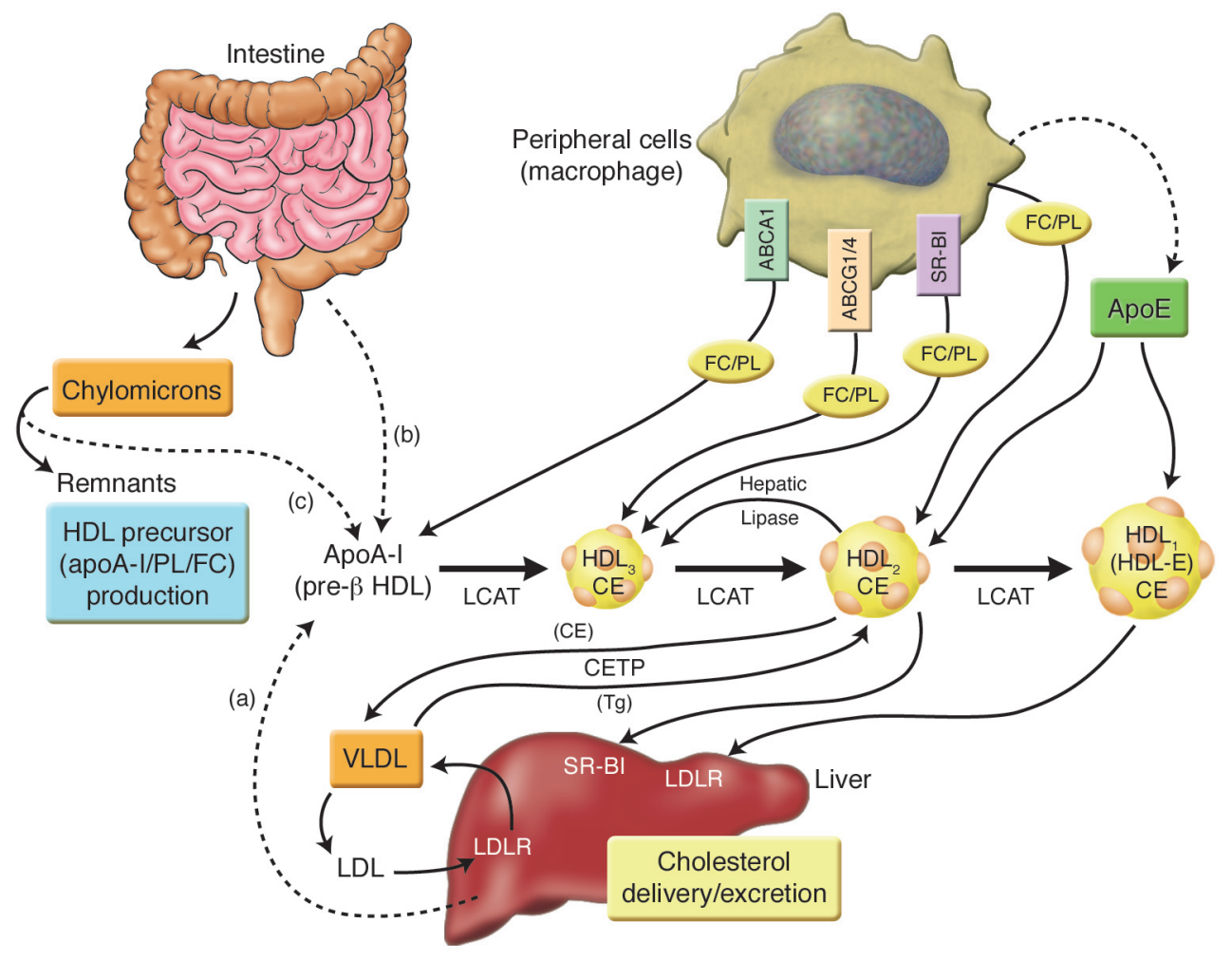

\section{Figure 1}

Role of HDL in the redistribution of lipids from cells with excess cholesterol (e.g., macrophages) to cells requiring cholesterol or to the liver for excretion. HDL precursors (pre- $\beta$ HDL) are produced by the liver and intestine (apoAI/PL/FC) or derived from surface material from chylomicrons after lipolysis $(a, b, c)$. Pre- $\beta$ HDL, $\mathrm{HDL}_{3}$, and $\mathrm{HDL}_{2}$ can accept cholesterol for reverse cholesterol transport. ApoE allows $\mathrm{HDL}_{2}$ particles to expand by enrichment with CE of the core after LCAT converts free cholesterol to $C E$. Larger HDL-with apoE $\left(\mathrm{HDL}_{2}\right.$ or $\mathrm{HDL}_{1}$ ) can deliver cholesterol to the liver directly via the LDL receptor. FC, free cholesterol; HDL-E, HDL-with apoE; LDLR, LDL receptor; PL, phospholipid; SR-BI, class $B$, type I scavenger receptor; $\mathrm{Tg}$, triglyceride. to CETP deficiency, the HDL-with apoE subclass is markedly increased, resulting in elevated HDL levels (20). Despite the potential benefit of such an antiatherogenic HDL profile in these subjects, there has been a conflict in the literature as to whether CETP deficiency is pro- or antiatherogenic in humans $(21,22)$. Thus, it is unclear whether the CETP inhibitors currently in clinical trials would promote or prevent atherosclerosis and coronary heart disease. Matsuura et al. (14) addressed an important aspect of this question and demonstrated that HDLwith apoE in CETP-deficient humans can accept unesterified cholesterol from macrophages and that LCAT esterified the cholesterol. This resulted in particle size expansion in the presence of apoE, which presumably could deliver this cholesterol directly to the liver.

\section{Importance of apoE for HDL particle expansion}

Previously, it was demonstrated that feeding a cholesterol-rich diet to swine resulted in large HDL-with apoE $(\sim 180 \AA)$ enriched in $C E$ that approached the size of LDL ( 220 $)$ (23, 24). Using a combination of calorimetry and small- angle $x$-ray scattering, it was shown that, compared with LDL, which contained 2 ordered layers of CE in its core, the relatively smaller swine HDL-with apoE contained a single order layer, accounting for the size difference (25). In both in vivo cholesterol feeding studies and in vitro studies of cholesterol loading, HDL-with apoE enlarged significantly through an increase in the number of ordered layers of CE within the core (140-180 A particles, 1 layer; 200-220 ̊, 2 layers; 240-260 ̊, 3 layers) $(26,27)$.

The importance of apoE in accepting cholesterol from cholesterol-loaded

\section{Figure 2}

Based on x-ray crystallographic data, apoE is envisioned to form 2 circular horseshoeshaped bands around a spherical phospholipid particle. Left: The light yellow core in the center represents the phospholipid hydrophobic core containing the fatty acyl chains; the outer light purple ring represents the phospholipid polar head groups. ApoE primarily interacts with the polar head groups, not the core acyl chains. Thus, apoE on a particle is uniquely capable of facilitating core expansion and accommodating increased CE content following LCAT activity. Right: Cross section of a region of the particle showing the relationship of the 2 apoE helices to the surface phospholipid head groups and core acyl chains.

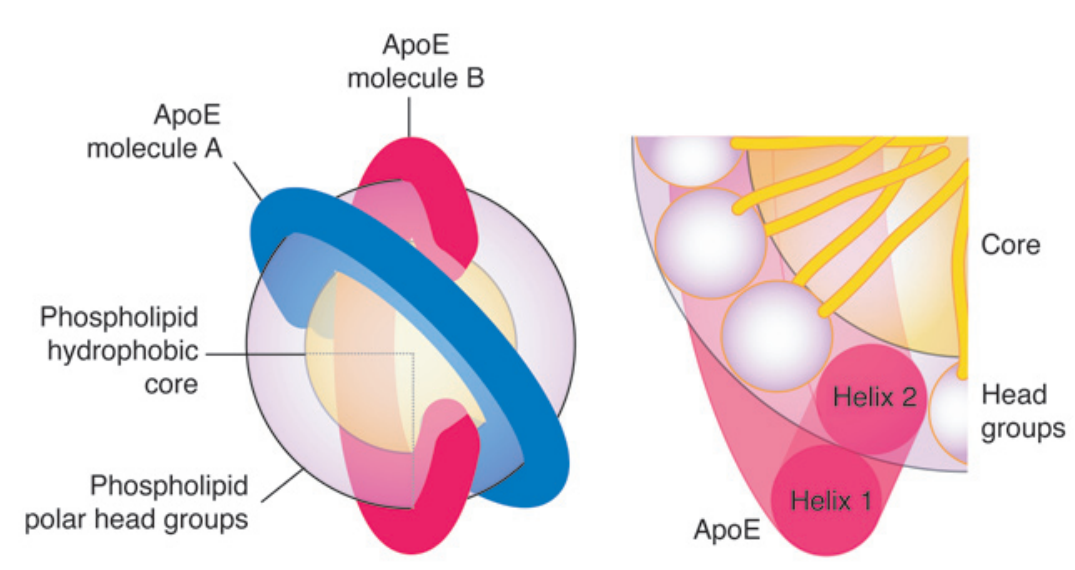


macrophages and facilitating the expansion of the CE core of HDL in conjunction with the action of LCAT was demonstrated in a series of studies with canine HDL. In one study, canine HDL-with apoE decreased CE formation and accumulation in cholesterol-loaded macrophages, reflecting enhanced cholesterol efflux from the cells; the efficiency of this effect was correlated with protection from atherosclerosis in animal models lacking CETP (28). In an in vitro study, when HDL in canine serum was incubated with cholesterol-celite particles or cholesterol-loaded macrophages as sources of cholesterol, the particles increased in size and CE content (26). The results were consistent with esterification of acquired cholesterol by LCAT and size expansion facilitated by apoE. A stepwise increase in the size of the HDL-with apoE was consistent with the addition of 1, 2, and 3 ordered layers of CE in the core. Another study demonstrated an obligatory role for cholesterol and apoE in the expansion of HDL (29). Incubation of apoEdepleted canine HDL in the presence of LCAT and cholesterol-loaded J774 macrophages, which do not synthesize apoE, did not result in significant size expansion of the HDL. However, adding exogenous apoE to the incubation resulted in HDL size expansion, CE accumulation, and enrichment in apoE. In addition, the LDL receptor-binding activity was proportional to the apoE content.

Matsuura et al. (14) extended those previous observations by demonstrating that several components of the reverse cholesterol transport pathway in CETP-deficient species, such as apoE and LCAT, also function in humans.

\section{Potential differences in how apoA-I and apoE bind to lipids}

The conclusion from previous studies and the study by Matsuura et al. in this issue (14) is that apoA-I-containing HDL can accommodate only a limited amount of CE in its core, resulting in a limited size expansion, whereas the size and CE content of the HDL can be significantly increased when apoE is present. This raises the question of whether apoE and apoA-I bind differently to lipid. Insight into this possibility comes from a recent low-resolution $\mathrm{x}$-ray crystal model of apoE bound to phospholipid (30). By analogy with apoA-I, where phospholipid complexes are discoidal and the apoA-I helices are wrapped around the edge of the lipid bilayer in a beltlike manner (31), the expectation was that apoE would bind similarly to a lipid. However, the apoE: phospholipid particles were spheroidal rather than discoidal, and the apoE molecule was folded in half, forming a circular horseshoe 2 helices thick and 1 helix wide and not a complete belt (Figure 2). The hydrophobic face of the amphipathic helices of apoA-I interacts with the fatty acid acyl chains of the phospholipids. In contrast, the polar faces of apoE interact with the polar phospholipid head groups. The hydrophobic faces of apoE are involved in helix:helix packing and thus do not interact with the acyl chains in the core. This different mode of interaction with the surface of the particle may allow apoE to more easily accommodate core expansion of HDL than apoA-I as CEs are formed by LCAT.

\section{Future for CETP inhibitors?}

Matsuura et al. (14) have shown that the HDL-with apoE from CETP-deficient humans can effectively accept unesterified cholesterol from macrophages and that apoE, in the presence of LCAT, promotes HDL size expansion. These findings, coupled with the previous in vitro studies and demonstration of effective reverse cholesterol transport in CETP-deficient animal models, suggest that CETP inhibitors are likely to be effective in generating an antiatherogenic HDL profile in humans. However, as pointed out by Matsuura et al., the answer awaits completion of ongoing clinical trials.

\section{Acknowledgments}

We thank Sally King for manuscript preparation, Stephen Ordway and Gary Howard for editorial assistance, and John C.W. Carroll for graphics.

Address correspondence to: Robert W. Mahley, Gladstone Institute of Neurological Disease, 1650 Owens Street, San Francisco, California 94158, USA. Phone: (415) 734-2000; Fax: (415) 355-0820; E-mail: rmahley@gladstone.ucsf.edu.

1. Linsel-Nitschke, P., and Tall, A.R. 2005. HDL as a target in the treatment of atherosclerotic cardiovascular disease. Nat. Rev. Drug Discov. 4:193-205.

2. Tall,A.R.,Jiang, X.-C., Luo, Y., and Silver, D. 2000. 1999 George Lyman Duff memorial lecture: lipid transfer proteins, HDL metabolism, and atherogenesis. Arterioscler. Thromb. Vasc. Biol. 20:1185-1188.

3. Lewis, G.F., and Rader, D.J. 2005. New insights into the regulation of HDL metabolism and reverse cholesterol transport. Circ. Res. 96:1221-1232.

4. Mahley, R.W., Weisgraber, K.H., and Farese, R.V., Jr. 2003. Disorders of lipid metabolism. In Williams textbook of endocrinology. 10th edition. P.R. Larsen, H.M. Kronenberg, S. Melmed, and K.S. Polonsky, editors. Saunders. Philadelphia, Pennsylvania, USA. 1642-1705.

5. Dullaart, R.P.F., and van Tol, A. 2001. Role of phospholipid transfer protein and pre $\beta$-high density lipoproteins in maintaining cholesterol efflux from Fu5AH cells to plasma from insulin-resistant subjects. Scand. J. Clin. Lab. Invest. 61:69-74.

6. Von Eckardstein, A., et al. 1996. Phospholipid transfer protein mediated conversion of high density lipoproteins generates pre $\beta_{1}$-HDL. Biochim. Biophys. Acta. 1301:255-262.

7. Guendouzi, K., et al. 1999. Biochemical and physical properties of remnant-HDL $\mathrm{H}_{2}$ and of pre $\beta_{1}$ HDL produced by hepatic lipase. Biochemistry. 38:2762-2768.

8. Barrans, A., et al. 1994. Hepatic lipase induces the formation of pre- $\beta$ high density lipoprotein (HDL) from triacylglycerol-rich $\mathrm{HDL}_{2}$. A study comparing liver perfusion to in vitro incubation with lipases. J. Biol. Chem. 269:11572-11577.

9. Francone, O.L., Royer, L., and Haghpassand, M. 1996 . Increased pre $\beta$-HDL levels, cholesterol efflux, and LCAT-mediated esterification in mice expressing the human cholesteryl ester transfer protein (CETP) and human apolipoprotein A-I (apoA-I) transgenes. J. Lipid Res. 37:1268-1277.

10. Yokoyama, S. 2005. Assembly of high density lipoprotein by the ABCA1/apolipoprotein pathway. Curr. Opin. Lipidol. 16:269-279.

11. Oram, J.F. 2000. Tangier disease and ABCA1. Biochim. Biophys. Acta. 1529:321-330.

12. Weisgraber, K.H., and Mahley, R.W. 1980. Subfractionation of human high density lipoproteins by heparin-Sepharose affinity chromatography. J. Lipid Res. 21:316-325.

13. Mahley, R.W., Innerarity, T.L., Bersot, T.P., Lipson, A., and Margolis, S. 1978. Alterations in human high-density lipoproteins, with or without increased plasma-cholesterol, induced by diets high in cholesterol. Lancet. 2:807-809.

14. Matsuura, F., Wang, N., Chen, W., Jiang, X.-C., and Tall, A.R. 2006. HDL from CETP-deficient subjects shows enhanced ability to promote cholesterol efflux from macrophages in an apoEand ABCG1-dependent pathway. J. Clin. Invest. 116:1435-1442. doi:10.1172/JCI27602.

15. Krieger, M. 1999. Charting the fate of the "good cholesterol": identification and characterization of the high-density lipoprotein receptor SR-BI. Annu. Rev. Biochem. 68:523-558.

16. Ha, Y.C., and Barter, P.J. 1982. Differences in plasma cholesteryl ester transfer activity in sixteen vertebrate species. Comp. Biochem. Physiol. B. 71:265-269.

17. Mahley, R.W., Innerarity, T.L., Rall, S.C., Jr., and Weisgraber, K.H. 1984. Plasma lipoproteins: apolipoprotein structure and function. J. Lipid Res. 25:1277-1294.

18. Mahley, R.W. 1985. Atherogenic lipoproteins and coronary artery disease: concepts derived from recent advances in cellular and molecular biology. Circulation. 72:943-948.

19. Mahley, R.W. 1988. Apolipoprotein E: cholesterol transport protein with expanding role in cell biology. Science. 240:622-630.

20. Yamashita, S., et al. 1990. Accumulation of apolipoprotein E-rich high density lipoproteins in hyperalphalipoproteinemic human subjects with plasma cholesteryl ester transfer protein deficiency. J. Clin. Invest. 86:688-695.

21. Curb, J.D., et al. 2004. A prospective study of HDL-C and cholesteryl ester transfer protein gene mutations and the risk of coronary heart disease in the elderly. 
J. Lipid Res. 45:948-953.

22. Moriyama, Y., et al. 1998. A low prevalence of coronary heart disease among subjects with increased high-density lipoprotein cholesterol levels, including those with plasma cholesteryl ester transfer protein deficiency. Prev. Med. 27:659-667.

23. Mahley, R.W., Weisgraber, K.H., Innerarity, T., Brewer, H.B., Jr., and Assmann, G. 1975. Swine lipoproteins and atherosclerosis. Changes in the plasma lipoproteins and apoproteins induced by cholesterol feeding. Biochemistry. 14:2817-2823.

24. Mahley, R.W., and Innerarity, T.L. 1977. Interaction of canine and swine lipoproteins with the low density lipoprotein receptor of fibroblasts as correlated with heparin/manganese precipitability. J. Biol. Chem. 252:3980-3986.
25. Atkinson, D., Tall, A.R., Small, D.M., and Mahley, R.W. 1978. Structural organization of the lipoprotein $\mathrm{HDL}_{\mathrm{c}}$ from atherosclerotic swine. Structural features relating the particle surface and core. Biochemistry. 17:3930-3933.

26. Gordon, V., Innerarity, T.L., and Mahley, R.W. 1983. Formation of cholesterol- and apoprotein E-enriched high density lipoproteins in vitro. J. Biol. Chem. 258:6202-6212.

27. Tall, A.R., Atkinson, D., Small, D.M., and Mahley, R.W. 1977. Characterization of the lipoproteins of atherosclerotic swine. J. Biol. Chem. 252:7288-7293.

28. Innerarity, T.L., Pitas, R.E., and Mahley, R.W. 1982. Modulating effects of canine high density lipoproteins on cholesteryl ester synthesis induced by $\beta$-very low density lipoproteins in macrophages. Possible in vitro correlates with atherosclerosis. Arteriosclerosis. 2:114-124.

29. Koo, C., Innerarity, T.L., and Mahley, R.W. 1985. Obligatory role of cholesterol and apolipoprotein $\mathrm{E}$ in the formation of large cholesterol-enriched and receptor-active high density lipoproteins. J. Biol. Chem. 260:11934-11943.

30. Peters-Libeu, C.A., Newhouse, Y., Hatters, D.M., and Weisgraber, K.H. 2006. Model of biologically active apolipoprotein $\mathrm{E}$ bound to dipalmitoylphosphatidylcholine. J. Biol. Chem. 281:1073-1079.

31. Davidson, W.S., and Silva, R.A.G.D. 2005. Apolipoprotein structural organization in high density lipoproteins: belts, bundles, hinges and hairpins. Curr. Opin. Lipidol. 16:295-300. 